\title{
Abordaje multimodalidad para la toma de decisiones en la patología aórtica inflamatoria
}

\author{
Jorge Casas* \\ Mariana Corneli** \\ Gabriel Perea**
}

Correspondencia

* Especialista en imágenes cardiovasculares no invasivas. Instituto CEDIC. Bahía Blanca. Argentina

** Especialista en imágenes cardiovasculares no invasivas. Instituto Cardiovascular de Buenos Aires. Argentina

\section{Recibido: 05/03/2020}

Aceptado: 09/10/2020

En línea: 31/12/2020

Citar como: Casas J, Corneli M, Perea G. Abordaje multimodalidad para la toma de decisiones en la patología aórtica inflamatoria. Rev Ecocar Pract (RETIC). 2020 (Dic); 3 (3): 16-18. doi: 10.37615/retic.v3n3a8.

Cite this as: Casas J, Corneli M, Perea G. Multimodality approach for decision-making in inflammatory aorticdisease. Rev Ecocar Pract (RETIC). 2020 (Dic); 3 (3): 16-18. doi: 10.37615/retic.v3n3a8.

\section{Palabras clave}

$\triangleright$ Aortitis

$\triangleright$ Aneurisma

$\triangleright$ Tomografía computada

$\triangleright$ Tomografía por emisión de positrones

\begin{tabular}{l} 
Keywords \\
\hline Aortitis \\
$\triangleright$ Aneurysm \\
$\triangleright$ Computed tomography \\
$\triangleright$ Positron emission tomography
\end{tabular}

\section{RESUMEN}

La inflamación aórtica (aortitis) suele ser consecuencia de vasculitis, principalmente autoinmunes, que en un alto porcentaje de los casos comprometen la aorta, con potenciales eventos graves o la necesidad de intervenciones asociadas. La presentación del siguiente caso tiene como objetivo conocer la prevalencia y el enfoque diagnóstico y terapéutico ante la sospecha de aortitis para acercarnos a saber cuál es el mejor manejo en estos pacientes.

\section{Presentación del caso}

Mujer de 72 años con antecedente de hipotiroidismo, obesidad, dislipidemia y diagnóstico 13 años atrás de arteritis de células gigantes (ACG) mediante biopsia de arteria temporal, por lo cual recibió tratamiento esteroide con buena respuesta al mismo.

Fue derivada al Servicio de Cardiología por el hallazgo de dilatación de la aorta torácica descendente $(40 \mathrm{~mm}$ ) y abdominal $(34 \mathrm{~mm})$ en una tomografía computarizada (TC) realizada por un episodio de dolor abdominal. En la primera consulta, la paciente se encontraba asintomática y no presentaba hallazgos patológicos al examen físico. Sin embargo, en el laboratorio de control presentaba una velocidad de eritrosedimentación notablemente aumentada $(65 \mathrm{~mm} / \mathrm{h})$.

Luego de 6 meses, se realizó un ecocardiograma que evidenció dilatación de la aorta ascendente con un diámetro máximo medido de $50 \mathrm{~mm}\left(26,3 \mathrm{~mm} / \mathrm{m}^{2}\right)$ (Figura 1), diámetros del ventrículo izquierdo y fracción de eyección normales y ausencia de valvulopatías significativas. También se repitió la TC de aorta, para evaluar los diámetros aórticos y la cinética de la dilatación aórtica, donde se evidenció un incremento significativo de los mismos, con valores absolutos indicativos de rápida tasa de crecimiento y necesidad de cirugía profiláctica (Figura 2 y Tabla 1).
Dado los antecedentes de ACG que presentaba la paciente, se evaluó el caso de manera multidisciplinaria, y, dada la alta probabilidad de inflamación activa a nivel del tejido aórtico que pudiese complicar el procedimiento quirúrgiCO, se decidió solicitar una tomografía por emisión de positrones (PET) con 18-fluor-desoxiglucosa (18 FDG) fusionada para identificar actividad inflamatoria activa a nivel de la aorta y sus ramas.

El estudio 18 FDG-PET/TC puso en evidencia focos de intensa acumulación del radio-trazador a nivel de la aorta ascendente, cayado aórtico, aorta torácica descendente proximal y ambas arterias subclavias; hallazgos indicativos de inflamación activa a nivel de estos segmentos vasculares (Figura 3).

Con estos resultados, y ante la necesidad de cirugía profiláctica por los diámetros aórticos encontrados, se tuvieron en consideración los reportes que existen en cuanto al tratamiento en este grupo de pacientes, donde es frecuente la evolución quirúrgica tórpida dada la presencia de tejidos friables que complican el procedimiento. Se decidió entonces iniciar tratamiento con esteroides y tocilizumab, para luego reevaluar la presencia de actividad inflamatoria y programar el tratamiento quirúrgico o endovascular.

Una vez completado el tratamiento, se realizó un nuevo estudio 18 FDG-PET/TC que mostró ausencia de actividad inflamatoria (Figura 4), por lo que se solicitaron exámenes preoperatorios para programar la intervención. 
- Casos clínicos caso 05

En los días siguientes, encontrándose en su domicilio, la paciente sufrió episodio de dolor torácico seguido de muerte súbita que no respondió a las maniobras de reanimación realizadas por el servicio de emergencia, ocurriendo exitus letalis.

\section{Estudio por imagen}

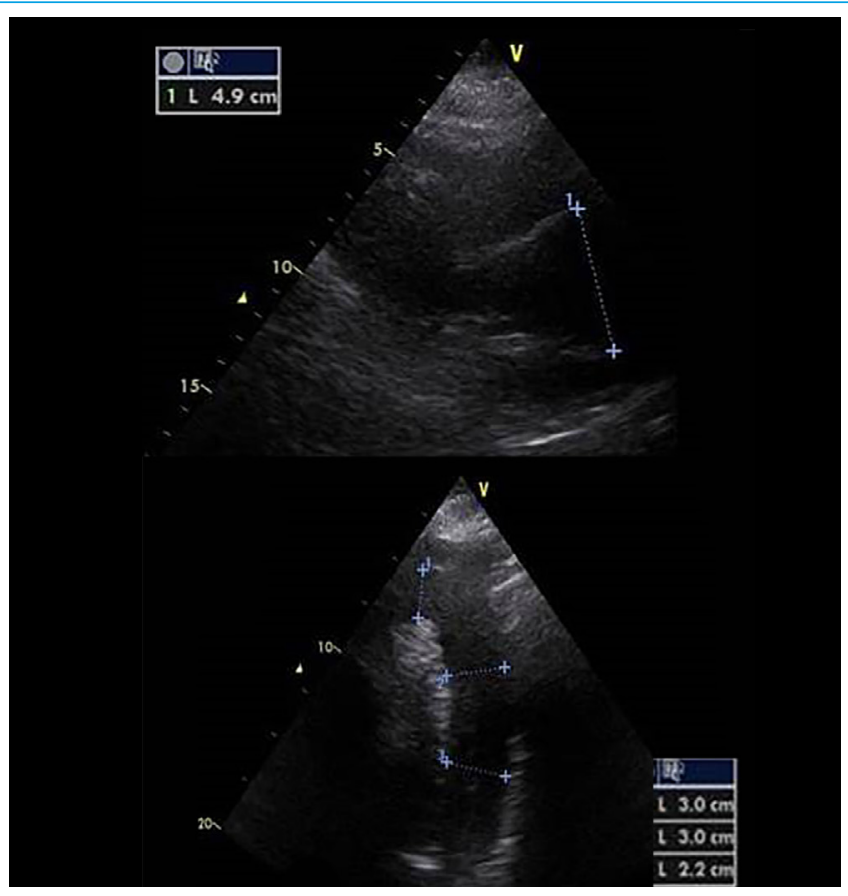

Figura 1. Ecocardiograma transtorácico, donde se evidencia dilatación y pérdida de la arquitectura de la aorta ascendente proximal (izquierda) y aorta torácica descendente proximal (derecha)

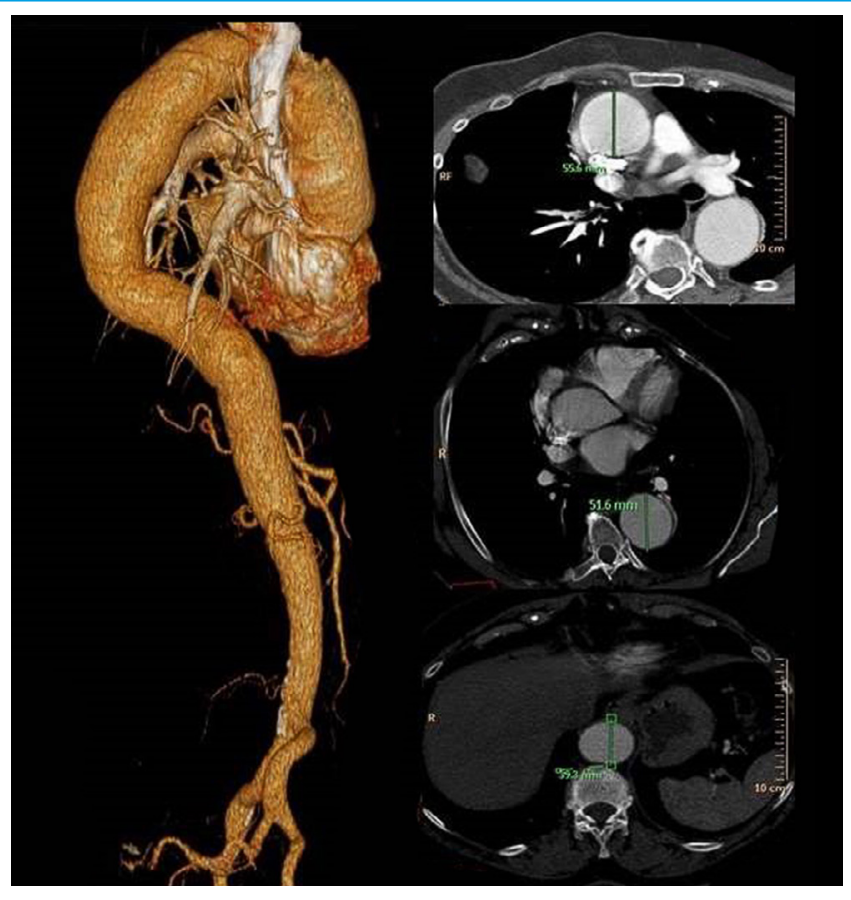

Figura 2. Angio-TC de aorta. En la izquierda, reconstrucción volumétrica tridimensional. En la derecha, cortes axiales a nivel de la aorta ascendente, descendente y abdominal, donde se observa el aumento de sus respectivos diámetros)

\begin{tabular}{|l|c|c|}
\hline \multicolumn{1}{|c|}{ Aorta } & Angio-TC basal & Angio-TC a los 6 meses \\
\hline Anillo & - & $22 \mathrm{~mm}$ \\
\hline Porción sinusal & - & $28 \mathrm{~mm}$ \\
\hline Unión sinotubular & - & $31,1 \mathrm{~mm}$ \\
\hline Aorta tubular & - & $55,6 \mathrm{~mm}$ \\
\hline Cayado aórtico & - & $33,4 \mathrm{~mm}$ \\
\hline Torácica descendente & $42,4 \mathrm{~mm}$ & $51,6 \mathrm{~mm}$ \\
\hline Abdominal suprarrenal & $33,7 \mathrm{~mm}$ & $37,2 \mathrm{~mm}$ \\
\hline Abdominal infrarrenal & $28,5 \mathrm{~mm}$ & $28 \mathrm{~mm}$ \\
\hline
\end{tabular}

Tabla 1. Comparación de la evolución de las medidas de la aorta

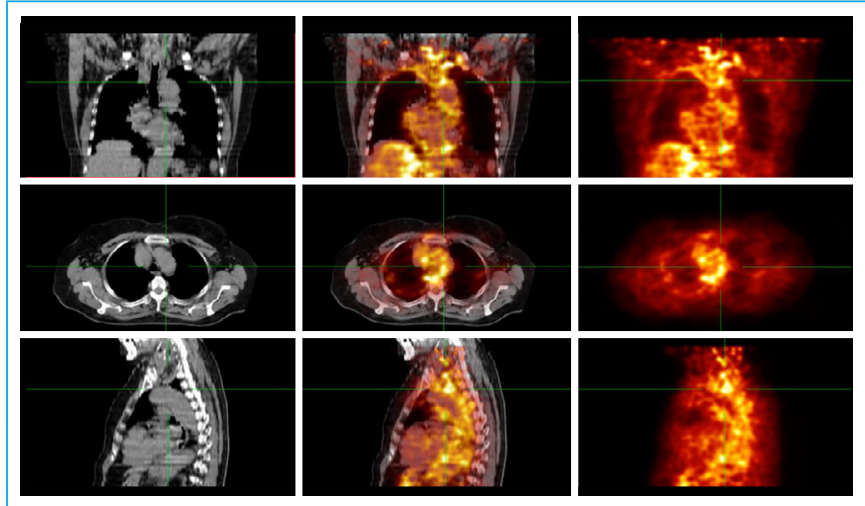

Figura 3.18 FDG-PET/TC. En la columna izquierda, cortes tomográficos (TC) En la columna central, imágenes de fusión (PET-TC), donde se identifica la intensa acumulación de la 18 FDG a nivel de la aorta ascendente, cayado aórtico, aorta descendente y ambas arterias subclavias. En la columna derecha, imágenes PET
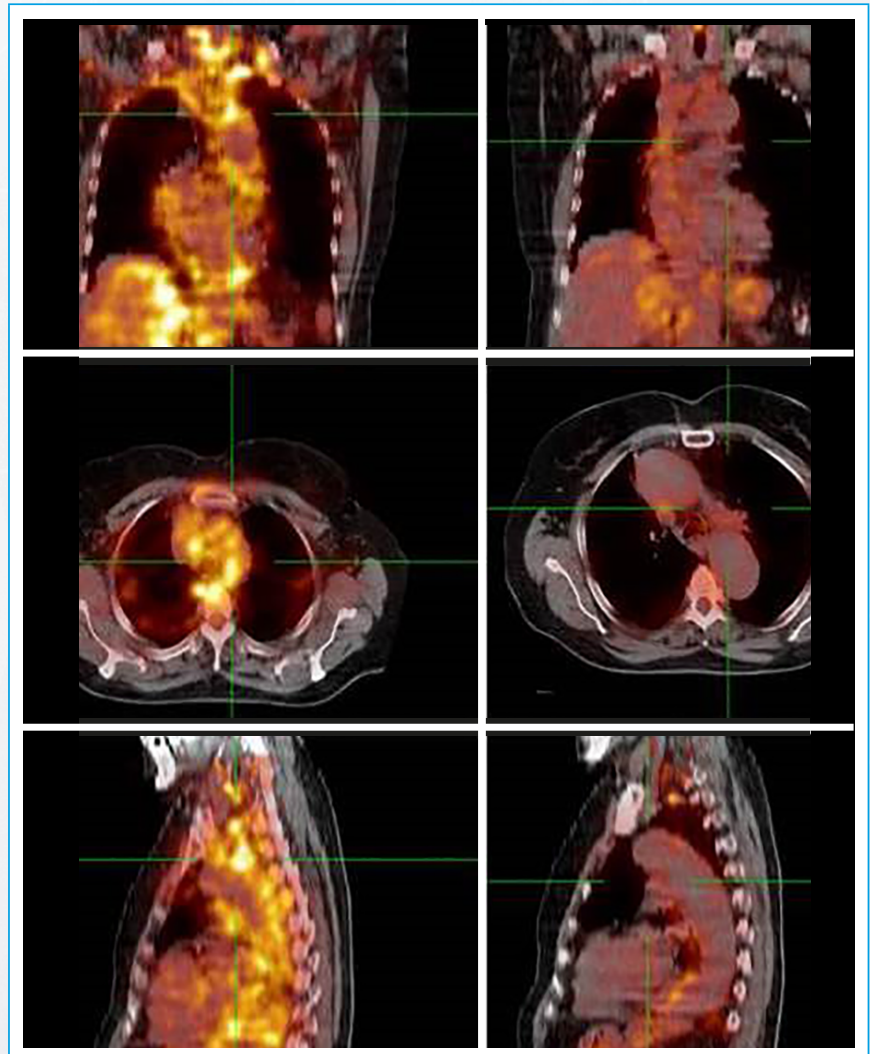

Figura 4. 118 FDG-PET/TC comparativo: previo al tratamiento con corticoides e inmunosupresores (izquierda) y posterior al mismo (derecha) 


\section{Discusión}

Habitualmente, entre las etiologías de las aortopatías, las enfermedades reumatológicas ocupan un importante lugar. El progreso de las técnicas de imágenes y el mayor conocimiento de las patologías reumáticas nos han permitido la detección precoz del compromiso aórtico potencialmente grave en estos pacientes. Se estima que, según el método de detección, la prevalencia de compromiso aórtico puede llegar al 50\% de los casos en pacientes con vasculitis ${ }^{(1)}$. En función de esto, en los últimos 2 años distintas sociedades científicas han redactado recomendaciones para el adecuado enfoque diagnóstico y terapéutico de estas patologías ${ }^{(2,3)}$.

Desde el punto de vista contextual, la aortitis se ubica dentro de las arteritis de grandes vasos, siendo sus principales entidades la arteritis de células gigantes (ACG) y la arteritis de Takayasu (AT $)^{(1,2)}$. También colagenopatías como el lupus eritematoso sistémico y la artritis reumatoidea pueden cursar con compromiso aórtico, así como otras causas no reumatológicas menos prevalentes (sífilis, tuberculosis...) ${ }^{(4)}$.

Desde el punto de vista clínico, la ACG afecta preferentemente a mayores de 50 años, con una relación entre sexo femenino y masculino de 2.5:1 (1,2). En el caso de la AT, afecta fundamentalmente a jóvenes menores de 40 años y la relación sexo femenino/masculino es de 9:1 ${ }^{(1,5)}$. En ambos casos, los síntomas y signos derivan principalmente del compromiso inflamatorio de los trayectos arteriales.

En cuanto al diagnóstico, en el caso de la ACG, históricamente se realizó mediante biopsia de la arteria temporal. En los últimos años se jerarquiza al ultrasonido (US), que se asocia a alta especificidad y sensibilidad para detectar compromiso inflamatorio característico a nivel de la arteria temporal y subclavia $^{(2,5)}$. En el caso de la AT, se debe comprobar el compromiso arterial proximal característico. Años atrás, se realizaba únicamente a través del estudio angiográfico invasivo; sin embargo, con el desarrollo de la angiotomografía computarizada (angio-TC) y la angio-resonancia magnética (angio-RM), estos métodos constituyen la forma de diagnóstico, recomendándose esta última por el no requerimiento de contraste ni radiaciones, sobre todo considerando que se trata de pacientes jóvenes.

Tanto en la ACG como en la AT, identificar y evaluar la aorta es mandatorio en estos pacientes, dadas las consecuencias que presenta el compromiso aórtico en forma de desarrollo de aneurismas y/o disección ${ }^{(4,5)}$. Se dispone para su exploración de ultrasonido, angio-TC, angio-RM o PET-TC; las últimas tres técnicas, además de evaluar los diámetros aórticos, también aportarán datos sobre e compromiso inflamatorio, siendo el PET-TC el método estándar para este fin ${ }^{(6)}$.

En cuanto al seguimiento, el intervalo de tiempo necesario para repetir los exámenes dependerá de los resultados iniciales. En casos de ausencia de compromiso aórtico, se podrá realizar un control más espaciado; y, si existiera compromiso inicial, se recomienda un seguimiento pautado de acuerdo con los diámetros detectados ${ }^{(4)}$.

Las indicaciones de intervención quirúrgica sobre la aorta en estos pacientes no varían respecto a la población general, es decir, cirugía de urgencia en caso de casos agudos y diámetros mayores a $55 \mathrm{~mm}$ o crecimiento mayor de 10

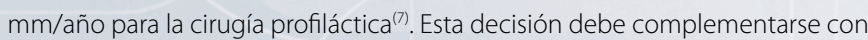
la valoración de la actividad inflamatoria a nivel de la aorta, como se comentó previamente.

\section{Conclusión}

La aortitis forma parte del escenario de las vasculitis de grandes vasos. Las causas más frecuentes dentro de este grupo son la ACG y la AT, pero también se debe tener en cuenta que puede estar presente en otras enfermedades reumatológicas.

Una vez realizado el diagnóstico de vasculitis de grandes vasos, se recomienda la búsqueda sistemática de compromiso aórtico y tomar conducta de acuerdo con los diámetros detectados. A diferencia de otras aortopatías, el carácter inflamatorio de estas entidades hace útil descartar el compromiso inflamatorio activo.

El caso presentado ejemplifica el uso de la metodología diagnóstica en esta patología que, aunque poco prevalente, tiene complicaciones sumamente graves.

\section{Ideas para recordar}

- La valoración de la aorta es mandatoria en las enfermedades reumatológicas, ya que su afección está presente en un elevado porcentaje de los casos, fundamentalmente en las arteritis de grandes vasos.

- El método de elección, de acuerdo con cada paciente, podrá ser ultrasonido, angio-TC, angio-RM o PET-TC; recordando que los tres últimos pueden además cuantificar los diámetros y valorar actividad inflamatoria, siendo el PET-TC el estándar para este último fin.

- El control de la actividad inflamatoria previa a una intervención programada puede acompañarse de mejores resultados de los procedimientos.

\section{Bibliografía}

1. Jennette J, Falk R, Bacon P, Basu N, Cid M, Ferrario F, et al. 2012 revised international Chapel Hill Consensus Conference nomenclature of vasculitides. Arthritis Rheum 2013; 65 (1): 1-11.

2. Dejaco C, Ramiro S, Duftner C, Besson F, Bley TA, Blockmans D, et al. EULAR recommendations for the use of imaging in large vessel vasculitis in clinical practice. Ann Rheum Dis 2018; 77: 636-643.

3. Sade L, Akdogan A. Imaging for screening cardiovascular involvement in patients with systemic rheumatologic diseases: more questions than answers. Eur Heart J-Cardiovascular Imaging 2019; 20: 967-978.

4. Bois J, Anand V, Anavekar N. Detection of inflammatory aortopathies using multimodality imaging. Circ Cardiovasc Imaging 2019; 12 (7): e008471.

5. Mackie S, Dejaco C, Appenzeller S, Camellino D, Duftner C, González-Chiappe $S$, et al. British Society for Rheumatology guideline on diagnosis and treatment of giant cell arteritis. Rheumatology 2020; 59: e1-e23.

6. Oyama-Manabe N, Yabusaki S, Manabe O, Kato F, Kanno-Okada H, Kudo K. IgG4-related cardiovascular disease from the aorta to the coronary arteries: multidetector CT and PET/CT. RadioGraphics 2018; 38: 1934-1948.

7. Erbel R, Aboyans V, Boileau C, Bossone E, Di Bartolomeo R, Eggebrecht H, et al. 2014 ESC Guidelines on the diagnosis and treatment of aortic diseases. Document covering acute and chronic aortic diseases of the thoracic and abdominal aorta of the adult The Task Force for the diagnosis and treatment of aortic diseases of the European Society of Cardiology (ESC). Eur Heart J 2014; 35: 2873-2926. 